\title{
Caractérisation et évaluation des vivriers dans un système d'exploitation agricole à base de coton dans le département de Sinématiali - Côte d'Ivoire
}

\author{
Nafan DIARRASSOUBA ${ }^{1 *}$, Souleymane SILUE ${ }^{1}$, Jésus INZA FOFANA ${ }^{1}$, Noel \\ DOUGBA DAGO $^{1}$, Marie Thérèse NDRI KOUAME ${ }^{2}$ et Edmond KOUABLAN KOFFI ${ }^{3}$ \\ ${ }^{1}$ UFR Sciences Biologiques/Université Peleforo Gon Coulibaly BP 1328 Korhogo, Côte d'Ivoire. \\ ${ }^{2}$ Centre de Recherche en Écologie (CRE), 08 BP 109 Abidjan 08, Côte d'Ivoire. \\ ${ }^{3}$ Laboratoire Central de Biotechnologies, Centre National de Recherche Agronomique (CNRA), \\ 01 BP 1740 Abidjan 01, Côte d'Ivoire. \\ *Auteur correspondant, E-mail: nafandiarra@yahoo.fr ; nafan.diarassouba@upgc.edu.ci
}

\section{RESUME}

La présente étude visait à caractériser les systèmes d'exploitation agricoles à base de coton, puis d'évaluer l'importance des vivriers dans ces systèmes. Elle a été conduite sur un échantillon de 40 exploitants agricoles répartis dans 8 villages de Sinématiali. Les résultats ont indiqué que les systèmes dominants sont le maïs (3,675 ha) et le coton ( 3,14 ha). Ensuite viennent, l'arachide (1,125 ha), le riz (0,906 ha) le haricot (0,306 ha), le mil (0,297 ha) et l'igname (0,194 ha). Par ailleurs, le compte d'exploitation révèle que les vivriers ont les marges nettes à l'hectare les plus élevés avec respectivement 245 727,5 FCFA, pour le riz ; 58 004,7 FCFA pour le maïs ; 209 738,4 FCFA pour l'arachide et 239472 FCFA pour le mil contre 5 085,1 FCFA pour le coton. L'analyse du ratio de la marge brute sur le coût variable totale montre que 1 FCFA investi sur les parcelles de vivriers rapporte respectivement 4,09 F; 1,54 F; 7,44 F et 12,09 FCFA pour le riz, le maïs, l'arachide et le mil contre 0,36 FCFA pour le coton. Aussi, cette étude indique que les vivriers contribuent à hauteur de $98 \%$, au revenu annuel du paysan contre $2 \%$ pour le coton. Malgré cette faible contribution du coton dans les revenus du paysan, sa culture permet aux exploitants d'accéder aux intrants et aux matériels agricoles modernes pour accroître les superficies et les rendements agricoles.

(c) 2014 International Formulae Group. All rights reserved.

Mots clés : Systèmes de culture, coton, vivriers, marge brute, Côte d'Ivoire.

\section{INTRODUCTION}

Dans les années 1990, deux événements majeurs ont marqué l'évolution des systèmes agricoles : la naissance de la notion de développement durable (Vivien, 2001); son intrusion dans le domaine de l'agriculture et la reconnaissance et la promotion d'une agriculture multifonctionnelle (Caron et al., 2008). La politique agricole de la Côte d'Ivoire ne reste pas en marge de ces deux événements car le secteur agricole constitue la base de l'économie. En effet, l'agriculture contribue à environ un quart à la formation du PIB et constitue la source de revenus de $2 / 3$ des ménages et procure environ $75 \%$ des recettes d'exportation non pétrolières (PND, 2011). Ce secteur comprend une agriculture vivrière qui 
contribue à hauteur de $17 \%$ du PIB et une agriculture d'exportation dont la part dans le PIB est de 5\%. Il représente encore davantage en termes d'emplois puisqu'il emploie 60 pour cent de la population active masculine et 75 pour cent de la population active féminine (PND, 2011 ; BAfD / OCDE, 2003).

Jusqu'au milieu de la décennie dernière, le coton jouait un rôle important dans l'économie agricole de la Côte d'Ivoire, bien qu'il ne vienne qu'en troisième position parmi les produits agricoles d'exportation, derrière le café et le cacao. La filière représentait en 2001 environ $7 \%$ des exportations du pays et générait un chiffre d'affaires en devises de l'ordre de 53 milliards FCFA. Par ailleurs, elle constituait le poumon de l'économie des campagnes du Nord de la Côte d'Ivoire, et faisait vivre directement 180000 producteurs, soit environ 2,5 millions d'habitants.

Mais la part du coton dans l'économie n'a pas cessé de diminuer depuis 2003, du fait de la baisse de la production et de celle des cours du coton. En effet, à cause du contexte économique actuel marqué par la hausse tendancielle des prix des facteurs de productions et l'instabilité du prix d'achat du coton-graine sur le marché national et mondial, la marge bénéficiaire du coton a connu une diminution continue (Donikpoho, 2012 ; Sigué, 2011). La culture du coton a perdu sa suprématie sur les cultures vivrières qui contribuent à l'amélioration des conditions socio-économiques des exploitants agricoles (Sigué, 2011).

Dans la plupart des systèmes à base de coton, on note de plus en plus une certaine complémentarité entre les cultures vivrières et le coton. Cependant, l'ampleur de cette complémentarité à toujours été sous estimée. Aussi depuis des décennies que cette situation perdure, très peu de travaux ont été entrepris pour quantifier l'apport des cultures vivrières dans le revenu annuel du paysan. En fait, l'accès aux intrants agricoles se fait à crédit au compte du coton mais une partie de ces intrants est destinée aux cultures vivrières (Donikpoho, 2012). Aussi, en cas de situation d'impayés à la vente du coton, les revenus générés par les vivriers contribueraient au recouvrement des frais des intrants agricoles (Sigué, 2011).

Cette étude avait donc pour objectif principal d'inventorier les différentes systèmes de culture à base de coton puis d'évaluer l'incidence financière des vivriers sur le revenu moyen annuel du paysan dans ces systèmes d'exploitation agricole.

\section{MATERIEL ET METHODES \\ Caractéristiques du site d'étude}

$\mathrm{Au}$ cours de la campagne cotonnière 2009 - 2010, des enquêtes sur les systèmes de production agricoles à base de coton ont été menées à Sinématiali dans le district des savanes au Nord de la Côte d'Ivoire (Figure 1). Sinématiali s'étend sur une superficie de $680 \mathrm{~km}^{2}$ avec une population essentiellement constituée de sénoufos. Les coordonnées géographiques de la localité de Sinématiali sont de $09^{\circ} 27^{\prime} 37,1$ ' latitude Nord et $005^{\circ} 38^{\prime} 83,2^{\prime}$ ' longitude Ouest à une altitude moyenne de 342 mètres et se situe à $35 \mathrm{Km}$ de Korhogo. Les amplitudes thermiques quotidiennes et annuelles y sont relativement importantes, de l'ordre de $20{ }^{\circ} \mathrm{C}$, le taux d'humidité, inférieur à celui du sud du pays, varie de $40 \%$ à $50 \%$. Le climat est de type soudanais caractérisé par deux saisons : l'une sèche, de novembre à avril, ponctuée par l'harmattan et une saison pluvieuse qui s'étend de mai à novembre. Cette zone est arrosée par le fleuve Bandama et les rivières Tine et Tielogo. La précipitation moyenne annuelle est de $1091 \mathrm{~mm}$ de pluie.

\section{Matériel}

La présente étude a porté sur des exploitations agricoles à base de coton et de vivriers (riz, arachide, maïs, mil, haricot, etc.). La répartition des exploitants selon le village est présentée dans le Tableau 1. 


\section{Méthodes d'étude}

\section{Méthodes d'échantillonnage}

Cet article présente les principaux résultats d'une étude menée en 2011- 2012 dont l'objectif a été de caractériser les systèmes d'exploitation agricoles à base de coton puis d'évaluer l'importance des vivriers dans ce système. Cette étude a comporté de nombreux entretiens semi-directifs auprès des acteurs de la filière coton. Elle a eu pour support une fiche d'enquête portant des questionnaires menés dans huit villages situés dans la zone d'intervention de la Société Compagnie Ivoirienne de Coton (COIC). Un échantillon de 40 exploitants agricoles répartis dans huit villages a été interrogé dans la zone de Sinématiali par un échantillonnage raisonné. Les villages ont été choisis en collaboration avec le conseillé agricole de la société cotonnière COIC qui est implantée dans la zone en tenant compte de leur accessibilité et surtout de la disponibilité des producteurs tout au long du processus d'enquête. Les exploitations agricoles retenues ont été classées selon la classification typologique A, B, C, D utilisée au Mali (Nubukpo et Sadio, 2005).

Une exploitation agricole de type A est une exploitation équipée qui dispose d'au moins deux paires de bœufs de labour, d'une charrue, d'un multiculteur, d'un semoir, d'une charrette (asine ou bovine) et d'un troupeau bovin d'au moins 6 têtes en plus des bœufs de labour. Une exploitation de type B est une exploitation qui dispose d'une unité d'attelage. Une exploitation de type $\mathrm{C}$ est une exploitation possédant une unité d'attelage incomplète mais qui a l'expérience de la culture attelée. Enfin, une exploitation de type $\mathrm{D}$ est une exploitation non équipée qui pratique la culture manuelle.

\section{Méthodes de collecte de données}

La première phase de cette recherche a été consacrée à la collecte d'informations déjà disponibles sur la filière coton, les modèles d'analyse sur les concepts évoqués dans notre étude et sur les potentialités de la région des savanes en particulier la zone de Sinématiali. Le choix de la zone a été motivé par le fait que la région des savanes participe pour plus de $50 \%$ dans la production nationale du coton, mais aussi par l'accessibilité des sites par les équipes de recherche. Les différentes informations ont été collectées à partir d'une fiche d'enquête portant diverses données sur l'exploitation du paysan. Pour chaque exploitant agricole échantillonné, les enquêtes ont consisté en la collecte d'informations sur l'identité de l'enquêté, sur les actifs dans l'exploitation, sur les techniques de culture, sur les cultures pratiquées, leur superficies et sur les intrants agricoles. Un autre volet consistait à déterminer les charges supportées par les producteurs et les marges nettes de chaque système. La rémunération de la main d'œuvre, le coût, les doses et quantités d'intrants chimiques, le coût des matériels agricoles et des équipements de travail serviront à évaluer les coûts de production pour chaque spéculation.

\section{Méthodes d'analyse des données}

Les données recueillies lors des différentes investigations ont été dépouillées à l'aide du logiciel Excel. Les statistiques élémentaires, les tests de corrélations et les différents graphes ont été obtenus à l'aide du logiciel XLSTAT 2007.6.

\section{Les éléments du compte d'exploitation}

Une analyse comptable a permis de mettre en évidence les caractéristiques d'un compte d'exploitation qui est la représentation de la situation annuelle des produits et des charges totales d'une exploitation agricole. Le compte d'exploitation organise les informations concernant les coûts et les produits liées aux activités techniques de production des différentes cultures.

\section{Valeur de la Production Totale (VPT)}

C'est le produit de la quantité totale produite et du prix de vente. Elle se définit comme étant le produit de la Quantité (Q) x 
par le prix (P). Pour la campagne 2011- 2012, les prix du coton ont été respectivement de 175 FCFA / kg et 145 FCFA / kg pour le coton $1^{\text {er }}$ choix et $2^{\text {ème }}$ choix. Mais pour cette étude, le prix du coton $2^{\text {ème }}$ choix a été pris en compte dans nos analyses. Les prix bords champ ont été utilisés pour les vivriers. $\mathrm{Au}$ moment de cette étude, il était de 210 FCFA/kg pour le riz, de $120 \mathrm{FCFA} / \mathrm{kg}$ pour le maïs, de $200 \mathrm{FCFA} / \mathrm{kg}$ pour l'arachide et de $225 \mathrm{FCFA} / \mathrm{kg}$ pour le mil.

\section{Coûts Variables Totaux (CVT)}

Ils concernent les coûts des intrants (engrais, herbicides, insecticides). Ces coûts varient en fonction de l'exploitation et ont été évalués en FCFA/ha.

\section{Coûts Fixes Totaux (CFT)}

Ils correspondent aux charges qui ne varient pas quel que soit le niveau de la production. Il s'agit essentiellement de l'amortissement du matériel.

\section{Marge Brute (MB)}

La marge brute se définit comme étant la différence entre la valeur de la production totale et les coûts variables totaux.

MB = VPT - CVT .

\section{Marge Nette (MN)}

C'est la différence entre la marge brute et les coûts fixes totaux : $\mathbf{M N}=\mathbf{M B}$ - CFT.

\section{Revenu Agricole Annuel (RAA)}

Le revenu agricole annuel d'une spéculation est le produit de la superficie moyenne de la spéculation et de son revenu net en FCFA/ha.

\section{Les ratios}

$\mathrm{Au}$ cours de cette étude, deux ratios essentiels ont été analysés. C'est le rapport des coûts variables sur la valeur de la production totale (CVT/VPT). Il détermine la pression relative des charges variables sur les productions des différentes spéculations. Le ratio de la marge brute sur le coût variable
(MB/CVT) définit ce que rapporte 1 FCFA investi dans la production d'une spéculation donnée.

\section{RESULTATS}

\section{Typologie des exploitants agricoles enquêtés \\ Âge moyen et nombre actifs travaillant dans les exploitations}

Les chefs d'exploitation enquêtés ont une moyenne d'âge de 34,07 ans comprise entre 18 et 49 ans et un écart type de 08,09. L'ensemble de ces chefs d'exploitations utilisent en moyenne 05 personnes actives dans leurs champs. Les différentes caractéristiques des données sur l'âge, le nombre d'épouses, le nombre d'enfants et le nombre d'actifs sont résumées dans le Tableau 2. La répartition selon l'origine de l'exploitant montre que les paysans échantillonnés sont composés de 87,09\% d'allochtones, $10 \%$ d'autochtones Nafara et 04,50\% d'étrangers de nationalité malienne.

L'analyse factorielle des correspondances (AFC) des données portant sur les caractéristiques des exploitants et celles des exploitations agricoles montre que les deux premières composantes expriment $67,97 \%$ des informations de base. Globalement, les paramètres liés aux exploitations (intrant et surfaces emblavées) sont corrélés à l'axe 1. Par ailleurs, l'âge des exploitants et la superficie du riz décrivent l'axe 2 mais sont opposés par rapport à l'axe 1.

\section{Typologie des exploitations agricoles}

La culture du coton tout comme celle des vivriers se pratique en général selon trois modes: la culture manuelle, la culture attelée, la culture motorisée. Mais dans cette étude, quatre types d'exploitations ont été mis en évidence. Mais la majorité des exploitations échantillonnées $(82,50 \%)$ est de type B. Ce type d'exploitation emploi en moyenne cinq actifs agricoles et deux paires de bœufs de culture attelée qui mettent en valeur en moyenne dix hectares (Tableau 3). 
Toutefois, la méthode d'agrégation en lien moyen de la Classification Ascendante Hiérarchique (CAH) a permis de regrouper les exploitants agricoles en trois groupes selon la valeur moyenne en production (Figure 3 ). Le groupe 2 renferme $55 \%$ des exploitants agricoles échantillonnés. Il a pour individu central Soro Lamine. Les caractéristiques moyennes de chacun des groupes sont mentionnées dans le Tableau 4.

\section{Caractérisation des systèmes de cultures}

Selon nos investigations, les principales cultures rencontrées dans les exploitations agricoles sont le coton, le riz, le maïs, l'arachide et le mil. Parmi toutes ces spéculations, ce sont le maïs et le coton qui occupent les plus grandes superficies exploitées avec respectivement 147 hectares et 125,50 hectares (Tableau 5). Globalement, les vivriers occupent 252,37 hectares, soit deux fois plus des surfaces emblavées que le coton (125,50 ha).

$\mathrm{Au}$ seuil de $5 \%$, le test de corrélation montre une corrélation significative entre le nombre de bœufs attelés et la surface moyenne emblavée. En effet, les coefficients de corrélation de Pearson et de détermination $\mathrm{R}^{2}$ sont respectivement de 0,56 et de 0,32 entre les deux variables. En revanche les points sont très dispersés dans le nuage de points entre le nombre d'actifs et la surface moyenne cultivée (Figure 4). Ce qui signifie que les corrélations de Pearson et de $\mathrm{R}^{2}$ sont non significatives entre ces deux paramètres.

\section{Analyse technico-économique des systèmes de culture \\ Point des intrants}

Les principaux intrants utilisés par les exploitants agricoles sont constitués d'engrais NPK, d'urée, d'herbicides et d'insecticides. Les différents intrants et les coûts afférents par hectare sont mentionnés dans le Tableau 6. L'analyse des résultats présentés dans le Tableau 6 montre que le coton est la culture qui nécessite le plus d'intrants (83422,9 FCFA /ha) contre 16794,5
FCFA/ha et 2628,6 FCFA/ha respectivement pour le riz et pour le maïs.

\section{Analyse financière des exploitations}

Pour comparer les systèmes de production entre eux, il est important d'évaluer leur performance économique. Pour cela, la valeur ajoutée nette et le revenu agricole ont été calculés pour chaque exploitation enquêtée. Afin de pouvoir comparer les différents systèmes, le revenu agricole à été ramené à la surface emblavée par exploitant échantillonné. On obtient donc la Figure 3 qui représente le revenu agricole par actif en fonction de la surface de chaque exploitation. L'analyse du compte d'exploitation montre que le coton est la culture qui présente la plus petite marge nette (Tableau 7).

\section{Calcul des ratios}

Les ratios des coûts variables sur la valeur de la production totale montre que les charges variables influencent significativement la production de coton $(73 \%)$ et que les cultures vivrières en dépendent moins (Tableau 8). Par ailleurs, Le ratio de la marge brute sur le coût variable est plus important pour les cultures vivrières comparativement au coton.

\section{Analyse de la rentabilité financière}

De l'analyse du compte d'exploitation, il ressort que le coton considéré $2^{\text {ème }}$ choix, présente même une marge nette positive (5085,1 FCFA). Toutefois, celle-ci est moins importante que celles de cultures vivrières telles que le riz, le maïs, l'arachide et le mil qui ont respectivement pour marges nettes 245 727,5 FCFA, 58 004,7 FCFA, 209 738,4 FCFA et 239472 FCFA.

Le riz procure plus de revenu net $(245$ 727,5 FCFA) aux exploitants agricoles. Ensuite viennent respectivement le mil (239 472 FCFA) et l'arachide (209 738,4 FCFA). Et enfin, le maïs qui procure moins de revenu aux paysans, une marge nette de $58 \quad 004,7$ FCFA/ha. 
Revenu agricole annuel et contribution des différentes spéculations

Il ressort de notre étude que c'est l'arachide qui pourvoit le revenu annuel le plus élevé avec 343970,97 FCFA/an comparativement au coton qui contribue à hauteur de 20086,14 FCFA/an (Tableau 9). Toutefois les revenus présentés dans le tableau ci-dessus intègrent aussi bien les productions autoconsommées que celles vendues pour les cultures vivrières.

Tableau 1 : Répartition des exploitants selon le village.

\begin{tabular}{lcc}
\hline Villages & Effectif par village & $\begin{array}{c}\text { Fréquence par modalité } \\
(\%)\end{array}$ \\
\hline Domorivogo & 09 & 22,50 \\
Donafologovogo & 05 & 12,50 \\
Karimvogo & 04 & 10 \\
Missolgopmvogo & 04 & 10 \\
Pelibivogo & 06 & 15 \\
Sotinvogo & 03 & 07,50 \\
Wolonvogo & 04 & 10 \\
Yapégovogo & 05 & 12,50 \\
Nombre d'observations & 40 & 100 \\
\hline
\end{tabular}

Tableau 2 : Nombre d'actifs et moyenne d'âge des chefs d'exploitation.

\begin{tabular}{lcccc}
\hline Statistiques & Age & $\begin{array}{c}\text { Nombre } \\
\text { Épouse }\end{array}$ & $\begin{array}{c}\text { Nombre } \\
\text { enfants }\end{array}$ & $\begin{array}{c}\text { Nombre } \\
\text { d'actifs }\end{array}$ \\
\hline Nb. d'observations & 40 & 40 & 40 & 40 \\
Minimum & 18 & 1 & 00 & 2 \\
Maximum & 49 & 4 & 14 & 17 \\
1er Quartile & 28,75 & 1 & 2,75 & 3 \\
Médiane & 33 & 2 & 4 & 5 \\
3ème Quartile & 40,25 & 2 & 7,25 & 6 \\
Moyenne & 34,07 & 1,97 & 5,12 & 5,25 \\
Variance (n-1) & 65,40 & 0,69 & 14,574 & 7,94 \\
Écart-type (n-1) & 8,09 & 0,83 & 3,82 & 2,82 \\
\hline
\end{tabular}


Tableau 3 : Répartition typologique des exploitations agricoles.

\begin{tabular}{ccccccc}
\hline $\begin{array}{c}\text { Type } \\
\text { d'exploitation }\end{array}$ & Effectif & $\begin{array}{c}\text { Nombre } \\
\text { d'épouses }\end{array}$ & $\begin{array}{c}\text { Nombre } \\
\text { d'enfants }\end{array}$ & $\begin{array}{c}\text { Nombre } \\
\text { d'actifs } \\
\text { agricoles }\end{array}$ & $\begin{array}{c}\text { Nombre } \\
\text { de Bæufs } \\
\text { attelés }\end{array}$ & $\begin{array}{c}\text { Surfaces } \\
\text { moyenne }\end{array}$ \\
\hline A & 01 & 04 & 09 & 17 & 06 & 12,25 \\
B & 33 & 02 & 04 & 05 & 04 & 10,01 \\
C & 4 & 02 & 11 & 05 & 02 & 08 \\
\hline D & 2 & 03 & 11 & 09 & 00 & 04 \\
\hline
\end{tabular}

Tableau 4 : Typologies des exploitants agricoles selon la valeur en production.

\begin{tabular}{lccccc}
\hline Groupe & VP coton & VP Riz & VP Maïs & $\begin{array}{c}\text { VP } \\
\text { Arachide }\end{array}$ & VP Mil \\
\hline $\begin{array}{l}\text { 1. (Coulibaly } \\
\text { Gninidjo) }\end{array}$ & 16163,35 & 149191,70 & 281737,11 & 419476,80 & 106907,14 \\
2. (Soro Lamine) & 18524,29 & 251578,15 & 215446,03 & 224719,71 & 2850,85 \\
3. (Soro mamadou) & 11441,47 & 215011,56 & 169180,37 & 148564,70 & 169626,00
\end{tabular}

Tableau 5 : Surfaces occupées par différentes cultures dans les exploitations agricoles.

\begin{tabular}{lccccc}
\hline Variables & Minimum & Maximum & Moyenne & $\begin{array}{c}\text { Écart- } \\
\text { type }\end{array}$ & Surface totale \\
\hline Sup Coton & 1 & 10 & 3,14 & 1,98 & $\mathbf{1 2 5 , 5}$ \\
Sup Riz & 00 & 2 & 0,90 & 0,39 & $\mathbf{3 6 , 2 5}$ \\
Sup Maïs & 1 & 8 & 3,67 & 1,50 & $\mathbf{1 4 7}$ \\
Sup Arachide & 00 & 04 & 1,121 & 0,791 & $\mathbf{4 5}$ \\
Sup Mil & 00 & 01 & 0,297 & 0,406 & $\mathbf{1 1 , 8 7}$ \\
Sup Haricot & 00 & 02 & 0,306 & 0,523 & $\mathbf{1 2 , 2 5}$ \\
\hline \multicolumn{1}{c}{ Sup : superficie } & & & & &
\end{tabular}

Tableau 6 : Évaluation du coût des intrants par hectare et par spéculations en FCFA/ha.

\begin{tabular}{lcccccc}
\hline Intrants & coton & riz & maïs & arachide & Mil & Haricot \\
\hline NPK & 41745 & 6670 & 18055 & 0 & 0 & 0 \\
Urée & 10972 & 2637,5 & 7490,5 & 0 & 0 & 0 \\
Herbicide & 11293 & 7487 & 6643,8 & 2628,6 & 0 & 0 \\
Insecticide & 19412,9 & 0 & 0 & 0 & 0 & 0 \\
\multicolumn{1}{c}{ Total } & $\mathbf{8 3 4 2 2 , 9}$ & $\mathbf{1 6 7 9 4 , 5}$ & $\mathbf{3 2 1 8 9 , 3}$ & $\mathbf{2 6 2 8 , 6}$ & $\mathbf{0}$ & $\mathbf{0}$ \\
\hline
\end{tabular}


Tableau 7 : Compte d'exploitation moyen pour un hectare de coton, de vivriers (riz, maïs, mil et arachide).

\begin{tabular}{lccccc}
\hline Rubriques & \multicolumn{5}{c}{ Cultures } \\
\cline { 2 - 6 } & Coton & Riz & Maïs & Arachide & Mil \\
\hline Rendement Moyen (KG /ha) & 1143 & 1552 & 1402 & 1280 & 1170 \\
Prix (2e choix) & 145 & 210 & 120 & 200 & 225 \\
VPT (FCFA/ha) & 165735 & 325920 & 168240 & 256000 & 263250 \\
Semence & 0 & 8775 & 2450 & 6670 & 3400 \\
NPK & 41745 & 6670 & 18055 & 0 & 0 \\
UREE & 10972 & 2637,5 & 7490,5 & 0 & 0 \\
Insecticides & 19412,9 & 0 & 0 & 0 & 0 \\
Main D'œuvre & 46.293 & 44987 & 35144 & 21878,6 & 15750 \\
Entretien De Traction & 1950 & 540 & 2410 & 1375 & 950 \\
Achat Pile & 1045 & 400 & 550 & 400 & 0 \\
CVT (FCFA/ha) & 121417,9 & 64009,5 & 66099,3 & 30323,6 & 20100 \\
MB (FCFA/ha) & 44317,1 & 261910,5 & 102140,7 & 225676,4 & 243150 \\
CFT (FCFA/ha) & 39232 & 16183 & 44136 & 15938 & 3678 \\
Amortissement & 39232 & 16183 & 44136 & 15938 & 3678 \\
MN (FCFA/ha) & $\mathbf{5 0 8 5 , 1}$ & $\mathbf{2 4 5 7 2 7 , 5}$ & $\mathbf{5 8 0 0 4 , 7}$ & $\mathbf{2 0 9 7 3 8 , 4}$ & $\mathbf{2 3 9 4 7 2}$ \\
\hline
\end{tabular}

Tableau 8 : Analyse de quelques ratios de la production de coton et de vivriers (riz, maïs, mil et arachide).

\begin{tabular}{llllll}
\hline & coton & riz & maïs & arachide & Mil \\
\hline MB/CVT & 0,36 & 4,09 & 1,54 & 7,44 & 12,09 \\
CVT/VPT & $73 \%$ & $19 \%$ & $39 \%$ & $11 \%$ & $7 \%$ \\
\hline
\end{tabular}

Tableau 9 : Récapitulatif des revenus annuels agricoles par spéculation.

\begin{tabular}{lccc}
\hline Spéculatic & $\begin{array}{c}\text { Surface moyenne/ } \\
\text { spéculation }\end{array}$ & $\begin{array}{c}\text { Revenu net en FCFA } \\
\text { /ha/spéculation }\end{array}$ & $\begin{array}{c}\text { Revenu annuel en FCFA/ } \\
\text { spéculation }\end{array}$ \\
\hline Coton & 3,95 & 5085,1 & 20086,14 \\
Riz & 1,15 & 245727,5 & 282586,62 \\
Maïs & 4,46 & 58004,7 & 258700,962 \\
Arachide & 1,64 & 209738,4 & 343970,97 \\
Mil & 0,43 & 239472 & 102972,96 \\
\hline
\end{tabular}




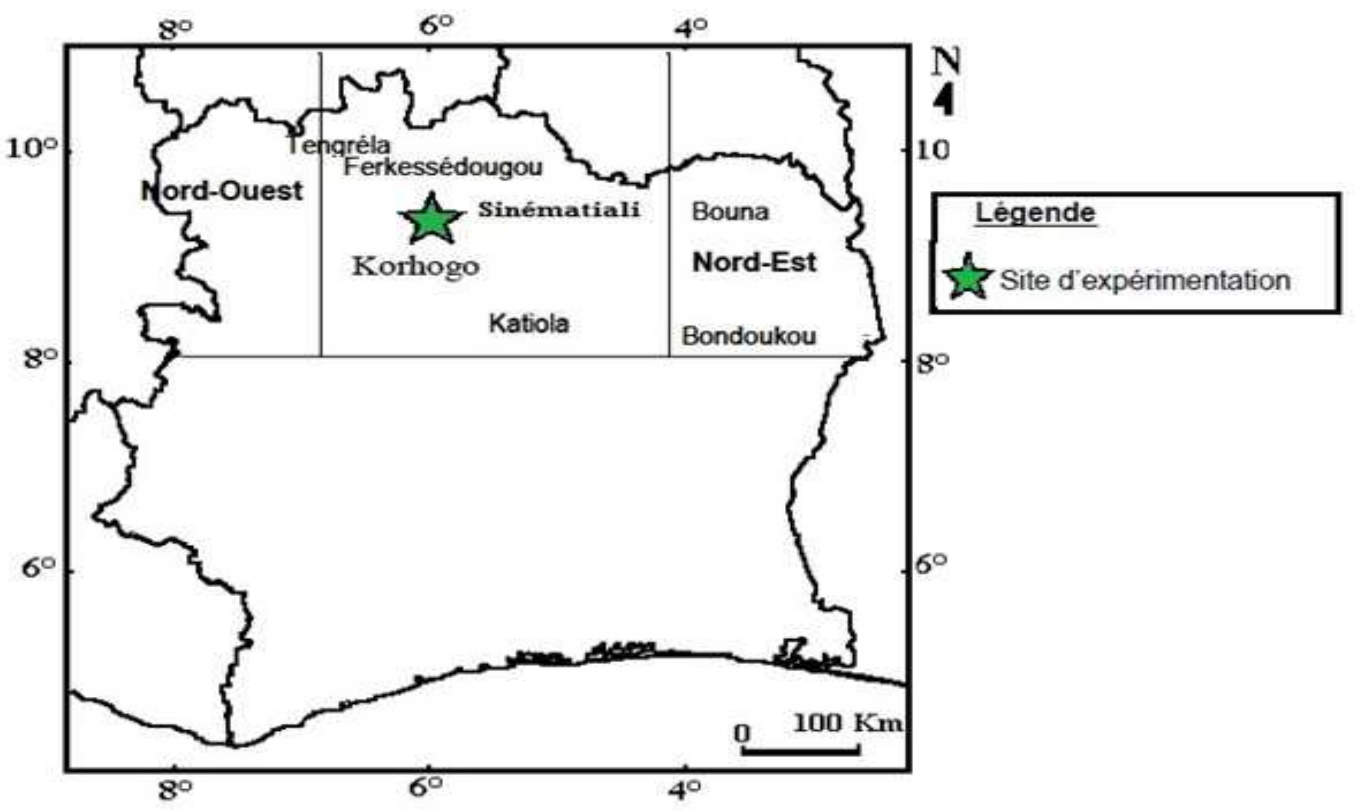

Figure 1 : Carte de la Côte d'Ivoire présentant le site d'étude.

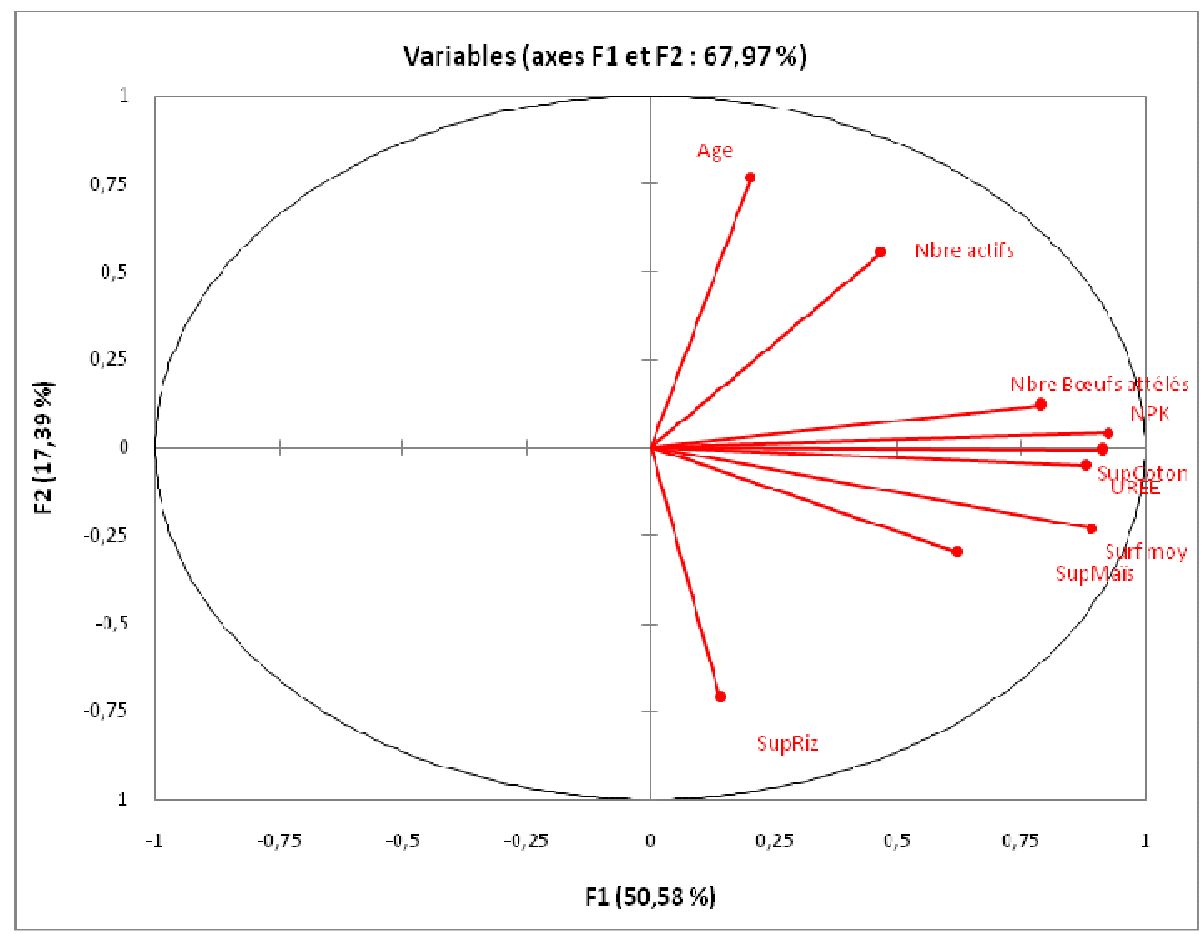

Figure 2 : Analyse factorielle des correspondances montrant les corrélations des axes 1 et 2 avec les caractéristiques des exploitants et celles des exploitations agricoles. 


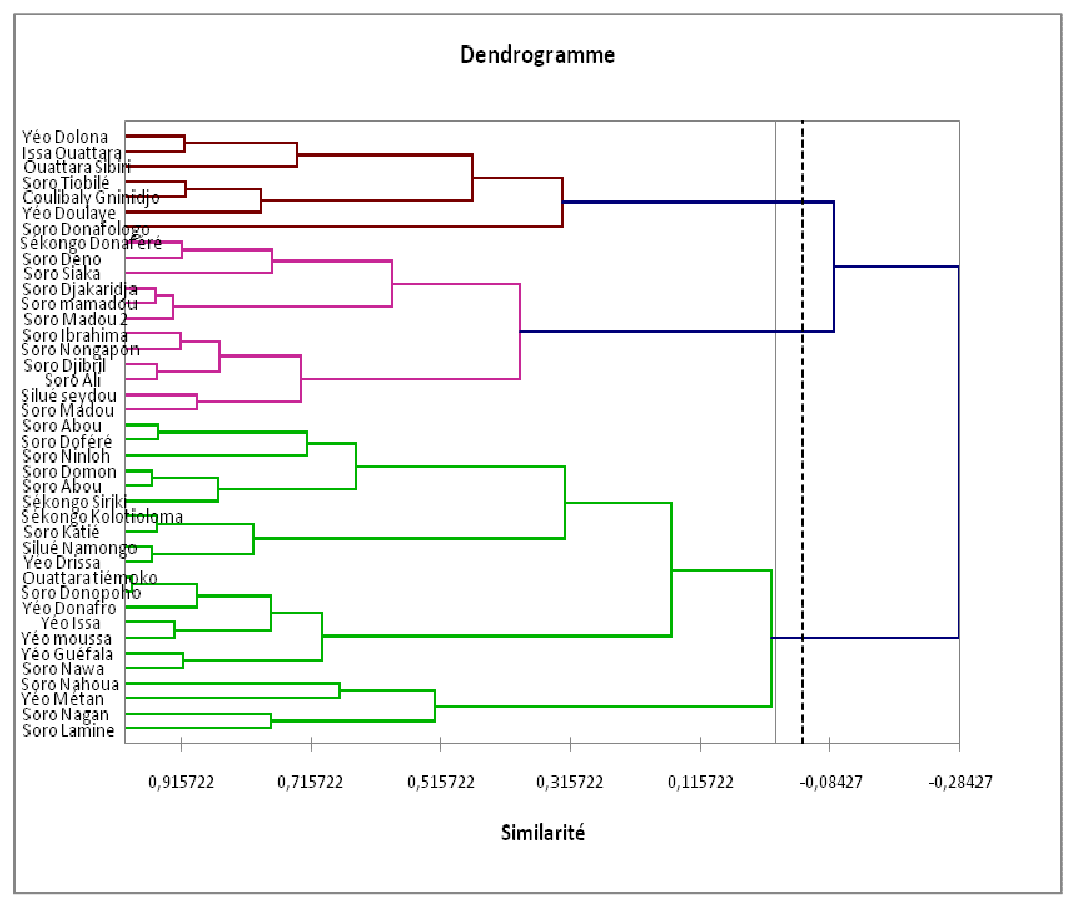

Figure 3 : Regroupement des exploitants agricoles par la méthode d'agrégation en lien moyen de la classification ascendante hiérarchique (Coefficient de corrélation de Pearson).

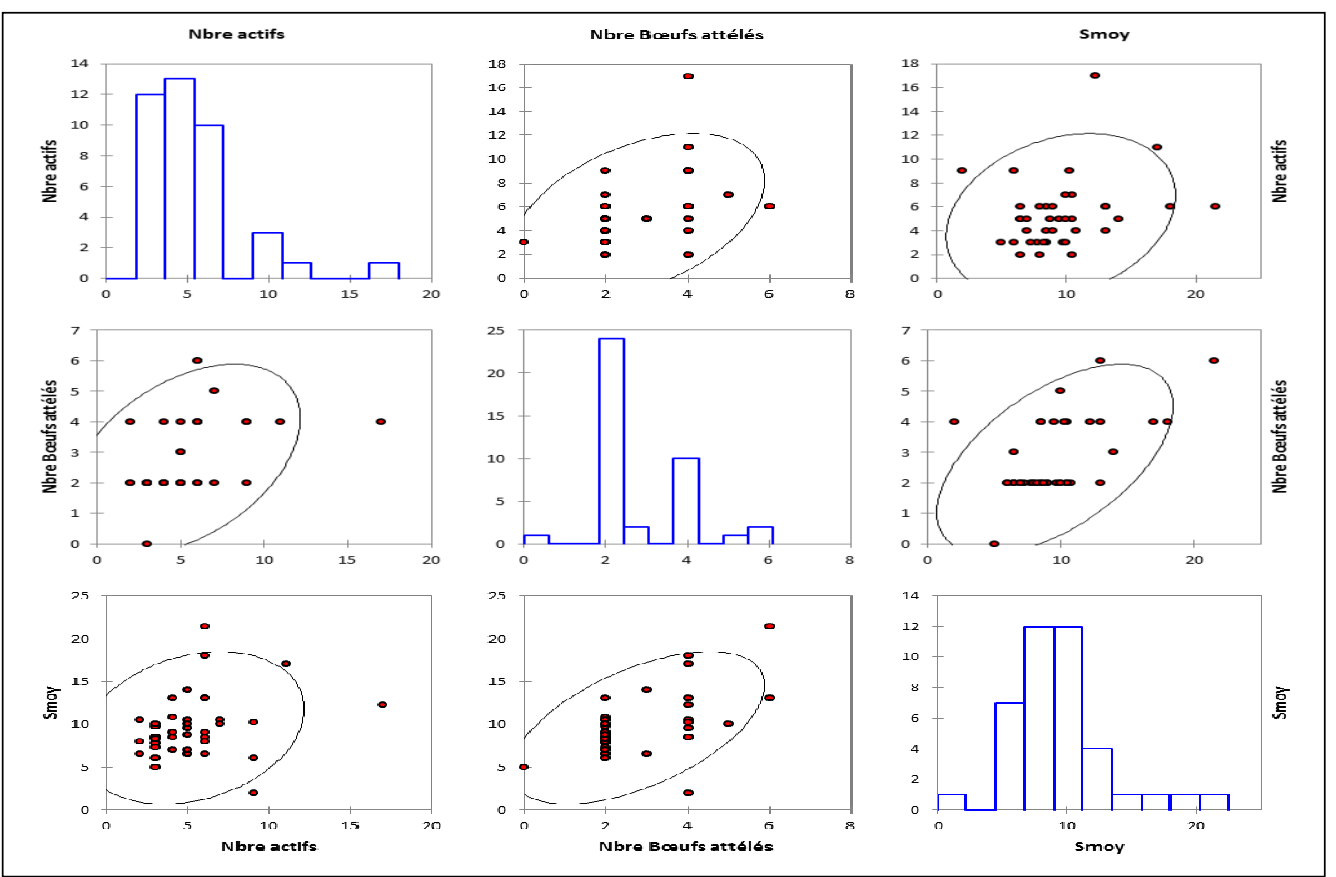

Figure 4 : Histogrammes et nages de points issus du test de corrélation de Pearson. 


\section{DISCUSSION}

Deux événements majeurs ont marqué l'évolution des systèmes agricoles : la naissance de la notion de développement durable (Vivien, 2001) et son intrusion dans le domaine de l'agriculture et la reconnaissance et la promotion d'une agriculture multifonctionnelle (Caron et al., 2008). L'évaluation de l'importance des vivriers dans un système à base de coton intervient dans un contexte économique délétère marqué par la l'instabilité du prix d'achat du coton graine sur le marché national et mondial. Ce qui a engendré une diminution continue de la marge bénéficiaire du coton. Les résultats ont indiqué que le maïs et le coton occupent les plus grandes superficies avec respectivement des taux de $36,93 \%$ et $32,66 \%$ des surfaces cultivées.

Le compte d'exploitation élaboré révèle que les vivriers ont les marges nettes à l'hectare les plus élevés avec respectivement 245 727,5 FCFA, pour le riz, 58 004,7 FCFA pour le maïs, 209 738,4 FCFA pour l'arachide et 239472 FCFA pour le mil. Ces valeurs sont nettement supérieures à celle du coton qui est seulement de 5085,1 FCFA. De cette analyse, il ressort que la marge nette par hectare obtenue pour une spéculation est d'autant moins importante que les coûts d'intrants nécessaires à la production de cette spéculation sont élevés. Ainsi, le coton qui nécessite les coûts variables les plus élevés (121 417,9 FCFA) procure le plus petit revenu net (5 085,1 FCFA). Ces informations mettent en évidence les effets positifs en termes de création de richesse et d'impulsion pour les autres cultures vivrières (arachide, mil etc.) qui utilisent peu ou pas d'intrants. On pourrait aussi expliquer cette situation par la forte dépendance du coton aux intrants, d'une part et au fait que les doses normales d'intrants agricoles destinées à la culture du coton ne sont pas apportées qu'aux cotonniers d'autre part. En effet, la majorité des paysans apportent une part importante des intrants destinés au coton à la fertilisation des champs de riz et de maïs. De manière synthétique, ces données tendent à montrer que le secteur $\mathrm{du}$ coton peut être un moteur pour le développement des autres cultures.

L'analyse des ratios confirme cette remarque. D'une part, le ratio du coût variable total sur la valeur totale de la production (CVT/VPT) révèle que pour obtenir 100 FCFA de valeur de production, l'exploitant investit en coût variable 19 FCFA pour le riz, 39 FCFA pour le maïs, 11 FCFA pour l'arachide, 7 FCFA pour le mil contre 73 FCFA pour le coton. Par ailleurs, le ratio de marge brute sur le coût variable total (MB/CVT) montre que pour 1 FCFA variable investi sur chaque spéculation, on gagne 4,09 FCFA pour le riz, 1,54 FCFA pour le maïs, 7,44 FCFA pour l'arachide, 12,09 FCFA pour le mil contre 0,36 FCFA pour le coton.

L'estimation des revenus agricoles annuels par spéculation montre que les vivriers rapportent à eux seuls plus de 988231,51 FCFA soit 98\% du revenu moyen annuel par paysan comparativement à celui du coton qui contribue à hauteur de $2 \%$ du revenu annuel (20086,14 FCFA). En fait, le coton reste une culture qui permet d'acquérir du matériel à crédit avec la société en charge de la filière. Dans la filière coton, l'acquisition des intrants agricoles et matériels nécessaires à l'activité de production se fait dans la majorité des cas à crédit. Les intrants chimiques et les herbicides sont généralement livrés à crédit aux producteurs. A la fin de la campagne, une déduction est faite sur la vente de la production du coton. Il peut arriver qu'à la fin de la campagne le producteur se retrouve en impayé, c'est-à-dire se retrouve avec un revenu brut qui ne peut pas couvrir la totalité des coûts des intrants acquit en début de campagne. C'est pourquoi certains agriculteurs le cultivent quelques années uniquement pour bénéficier des crédits d'achat de matériel.

De l'analyse de la littérature existante, la culture du coton induit un effet positif sur les productions vivrières, en particulier à travers l'arrière-effet positif de l'engrais coton sur le rendement des cultures vivrières. Une 
illustration de cette caractéristique est donnée par les résultats de recherches menées par FAO/ICRISAT (1996). De cette recherche, l'analyse comparative des rendements observés sur les différentes cultures céréalières dans les exploitations productrices et non productrices de coton montre que dans les exploitations cotonnières, les rendements des céréales sont supérieurs à ceux des exploitations non cotonnières. L'augmentation de rendements dans les systèmes à base de coton est très importante par rapport aux rendements obtenus dans les systèmes de cultures céréalières pures (FAO, 1987). Cette différence entre les rendements s'expliquerait par la relative intensification observée dans le système de culture à base de coton et le système de rotation coton-céréales pratiqué par les producteurs de coton. Cette pratique permet aux céréales de bénéficier des arrièreseffets de l'engrais coton.

Par ailleurs, outre le niveau de connaissance des producteurs, la question de leur capacité à soutenir l'investissement que représente, à chaque campagne, la question des intrants agricoles est source d'inquiétudes dans la mesure où les résultats présentés dans cet article soulignent le peu de bénéfice économique retiré par les producteurs de coton (Renaudin et al., 2012). Surtout, ils laissent entrevoir le risque financier que comportent les crédits pour l'achat des intrants pour ceux qui sont financièrement moins nantis, obtiennent des rendements insuffisants pour supporter le coût de production. Celui-ci pourrait d'ailleurs contribuer à fragiliser davantage ce type d'agriculteurs, dans un contexte ou les rendements demeurent encore largement tributaires des aléas pluviométriques (Vitale et al., 2008). Le danger que leur revenu chute et, qu'ils ne parviennent pas à rembourser les crédits pour l'achat des intrants qu'ils ont contractés auprès de la société cotonnière, est grand.

Toutefois, malgré le faible revenu annuel généré par le coton, sa culture permet aux paysans de s'approvisionner en intrants agricole aussi bien pour le coton que pour les cultures vivrières dont l'apport d'intrant est indispensable. L'introduction de la culture du coton a permis aussi aux paysans de passer du système de type traditionnel, au système de production moderne. Elle a surtout permis une évolution de la mentalité du paysan, qui prend en compte la notion de rentabilité et la maîtrise des techniques culturales.

\section{Conclusion}

$\mathrm{Au}$ terme de cette étude, on note que les cultures vivrières occupent une place importante dans le système agricole à base de coton. En matière de surfaces cultivées, les vivriers occupent $67,34 \%$ des superficies disponibles contre $32,66 \%$ pour la culture du coton. Il ressort aussi de cette étude que c'est le coton qui procure aux exploitants agricoles la plus petite marge nette (5085,1 FCFA/ha) car il occasionne les coûts variables les plus élevés (121417,9 FCFA/ha). L'analyse financière indique que les cultures vivrières fournissent aux paysans $98 \%$ de leur revenu moyen agricole annuel. Pour une meilleure promotion des cultures vivrières dans le système à base de coton, les agents d'encadrement (conseillers agricoles) devraient sensibiliser et former les paysans aux nouvelles techniques culturales disponibles comme ils le font pour le coton. $\mathrm{Vu}$ les revenus agricoles générés par les vivriers, il est souhaitable que les sociétés cotonnières promeuvent les cultures vivrières en favorisant l'accès aux intrants pour le riz et le maïs. Cela normaliserait les doses d'intrant apportées au coton et par ricochet améliorerait les rendements et les revenus annuels agricoles du coton. Tout cela ne peut être réalisable qu'avec une réorganisation des organisations professionnelles agricoles en micro entreprise de prestation. La prise en compte de toutes ces recommandations pourrait permettre aux paysans et à la COICSA d'améliorer les rendements agricoles puis contribuer à la réduction de la pauvreté qui s'inscrit dans la politique d'autosuffisance alimentaire à laquelle aspire la Côte d'Ivoire. 


\section{REMERCIEMENTS}

Les auteurs témoignent leur gratitude à Monsieur KONE Sigue pour le rôle joué dans la conduite des essais et la collecte des données sur le terrain.

\section{REFERENCES}

AGRER. 2007a. Etude sur l'analyse et les stratégies de développement durable de la filière coton. Version intermédiaire. P.147.

AGRER. 2007b. Stratégies de développement durable de la filière coton au Burkina Faso [en ligne]. Version amendée, P.60.

BAfD/OCDE. 2003. Perspectives économiques en Afrique pp 139-150.

Caron P, Reig E, Roep D, Hediger W, Le Cotty T. 2008. Multifunctionality: refocusing a spreading, loose and fashionable concept for looking at sustainability. Int. J. Agricultural Resources, Governance and Ecology, 7(4/5): 301-315.

Donikpoho K. 2012. Evaluation de l'importance des vivriers dans le système d'exploitation agricole à base de coton : cas des sections de Sinématiali et de Sédiogo. Mémoire de fin d'étude Licence 3. Université Peleforo Gon Coulibaly de Korhogo, Côte d'Ivoire, P 40.

FAO/ICRISAT. 1996. The World Sorghum and Millet Economies. FAO: Rome, Italy ; p.60.

FAO. 1987. Amélioration et production du maïs, du sorgho et du mil, volume 2 , sélection agronomie et production de semences. FAO, Rome, Italie, p.559.

Nubukpo KK, Sadio MK. 2005. L'impact sur l'économie malienne du nouveau mécanisme de fixation du prix du coton graine. Rapport d'activité, p.42.
PND. 2011. Plan National de Développement: Résumé Analytique Tome 1. PND, p.50.

Rouanet G. 1997. Le Maïs. Maisonneuve et La Rose, le Technicien d'Agriculture Tropicale : Paris ; 119.

Renaudin C, Pelc H, Opois J. 2012. Cotonnier génétiquement modifié : l'expérience d'une campagne agricole au Burkina Faso. Cah Agric, 21(6): 387-394.

Schilling R. 1996. L'Arachide. Maisonneuve et La Rose, le Technicien d'Agriculture Tropicale : Paris ; 27.

Sement G. 1996. Le Cotonnier en Afrique Tropicale. Maisonneuve et La Rose, le Technicien d'Agriculture Tropicale: Paris ; 17.

Sigue K. 2011. Evaluation de l'importance des vivriers dans le système d'exploitation agricole à base de coton : cas des sections de Derrière fleuve et de Nangounonkaha. Mémoire DUT, URES de Korhogo, Université de Bouaké, Côte d'Ivoire ; 39 .

Soffo MJJ. 2002. Monographie du secteur agricole de la région des savanes. Mémoire DUT, URES de Korhogo, Université de Bouaké, Côte d'Ivoire, p.36.

Vitale J, Glick H, Greenplate J, Traore O. 2008. The economic impacts of second generation Bt cotton in West Africa: empirical evidence from Burkina Faso. Int. J. Biotechnology, 10(2): 167-183.

Vivien FD. 2001. Histoire d'un mot, histoire d'une idée : le développement durable à l'épreuve du temps. In: Le Développement Durable, de l'Utopie au Concept : de Nouveaux Chantiers pour la Recherche, Marcel Jollivet (ed). Nature Sciences Sociétés, Elsevier ; 19-55. 